\title{
A COMPUTATIONAL METHOD FOR A CLASS OF JUMP LINEAR QUADRATIC SYSTEMS
}

\author{
K. H. WONG, N. LOCK and K. KAJI ${ }^{1}$
}

(Received 8 June 1993; revised 27 October 1993)

\begin{abstract}
A class of linear systems subject to sudden jumps in parameter values is considered. To solve this class of stochastic control problem, we try to seek the best feedback control law depending only on the measurable output. Based on this idea, we convert the original problem into an approximate constrained deterministic optimization problem, which can be easily solved by any existing nonlinear programming technique. An example is solved to illustrate the efficiency of the method.
\end{abstract}

\section{Introduction}

In $[2,3]$, the study of optimal control for a class of linear systems with random parameters was initiated. The class of stochastic optimal control problems considered in these references involved a jump linear quadratic (JLQ) system, where the parameters are Markov jump processes with finitely countable states, and where an optimal control law is sought with respect to the mathematical expection of a quadratic cost. In $[8,9,10]$, it was discovered that the optimal control law for this type of problem consisted of a full state feedback regulator. In [4], the optimal control law for the linear quadratic (LQ) problem without jumps in the parameter was established. In [7], the linear quadratic Gaussian (LQG) problem, where the parameters were subjected to continuously acting disturbances modelled by Gaussian white noise, was considered. In [6], the optimal feedback control law depending only on measurable output rather than on the state of the system for the JLQ problem was established. This result was further improved when a JLQG regulator was designed in [5] where only part of the system state, the output, was measured.

This paper deals exactly with the same problem as that considered in [6]. By seeking the best feedback control law depending only on the measurable output, we

\footnotetext{
'Dept of Computational and Applied Math., Univ. of the Witwatersrand, Johannesberg, South Africa.

(C) Australian Mathematical Society, 1995, Serial-fee code 0334-2700/95
} 
convert the original problem into an equivalent standard constrained deterministic optimization problem, which can be easily solved by any nonlinear programming technique. This method is better than those considered in [4] and [6] which are based on the necessary condition of optimality, because their algorithms could not guarantee that the system would be stable in every mode during each iteration of the algorithm. As in [1], the gradients of both the performance index and the constraints are derived via the introduction of the Hamiltonian and co-state matrices. Finally, an example is solved to illustrate the efficiency of our method.

\section{Statement of the problem}

Consider the following jump linear system

$$
\left\{\begin{array}{l}
\dot{x}(t)=A(r(t)) x(t)+B(r(t)) u(t) \\
y(t)=C(r(t)) x(t)
\end{array}\right.
$$

where $x(t) \in R^{n}$ is the state vector, $u(t) \in R^{m}$ is the control vector and $y(t) \in R^{r}$ is the output vector. The entries of the matrices $A(r(t)), B(r(t))$ and $C(r(t))$ are random, because they depend on the system mode $r(t) . r(t)$ is a stochastic jump process with a finite valuation set $S=\{1, \ldots, N\}$. The dynamics of $r(t)$ are given in terms of the transition probabilities

$$
\operatorname{Prob}\{r(t+\Delta)=j \mid r(t)=i\}= \begin{cases}\pi_{i j} \Delta+O(\Delta) & \text { if } i \neq j \\ 1+\pi_{i i} \Delta+O(\Delta) & \text { if } i=j\end{cases}
$$

The initial mode $r\left(t_{0}\right)$ is assumed to have a known distribution $P_{0}$.

For the sake of simplicity, let $\left[A_{i}, B_{i}, C_{i}\right]$ denote the matrices $[A(r(t)), B(r(t))$, $C(r(t))]$ when the system operates in the $i$ th mode (that is, $r(t)=i$ ).

We now define a quadratic performance criterion as follows:

$$
\bar{J}\left(u, t_{0}, x\left(t_{0}\right), r\left(t_{0}\right)\right)=E\left\{\frac{1}{2} \int_{t_{0}=0}^{\infty}\left[x(\tau)^{\top} Q x(\tau)+u(\tau)^{\top} R u(\tau)\right] d \tau \mid\left(t_{0}, x\left(t_{0}\right), r\left(t_{0}\right)\right)\right\}
$$

where $E$ stands for the mathematical expectation and $Q$ and $R$ are, respectively, a semipositive and a positive symmetric weighting matrix.

From [8] and [10], it is clear that the optimal control $u^{*}$ can be expressed in a closed-loop form

$$
u^{*}(t)=U(x(t), r(t)),
$$

with gains involving a set of $N$ coupled Riccati equations. However, in most problems, some of the required states or mode variables are not available to the designer. Thus, 
as mentioned in [6], the more practical control law is the linear output feedback mode dependent law, that is, the law

$$
u^{*}(t)=-F(r(t) y(t))
$$

which minimizes (3).

\section{An approximate problem}

First, we restrict the control to have the form of linear output feedback mode

$$
u(t)=-F_{i} y(t) \quad \text { when } r(t)=i,
$$

where $F_{i}(i=1, \ldots, N) \in R^{m \times r}$ is the gain matrix when the system is operating in mode $i$.

THEOREM 3.1. Assume that the system starts in mode $i_{0}$ and the initial state is $x_{0}$. Then the performance criteria (3) can be reduced to

$$
\bar{J}=\frac{1}{2} x_{0}^{\top} K_{i_{0}} x_{0},
$$

where $K_{i} \in R^{n \times n}$ (when $r(t)=i$ ) satisfies the equation

$$
K_{i} \tilde{A}_{i}+\tilde{A}_{i}^{\top} K_{i}+Q+C_{i}^{\top} F_{i}^{\top} R F_{i} C_{i}+\sum_{j=1}^{N} \pi_{i j} K_{j}=0, \quad i=1, \ldots, N
$$

where

$$
\tilde{A}_{i}=A_{i}-B_{i} F_{i} C_{i}, \quad i=1, \ldots, N,
$$

provided that the matrices

$$
\tilde{A}_{i}=\left(\tilde{A}_{i}+\frac{1}{2} \pi_{i i} I\right), \quad i=1, \ldots, N
$$

are stable.

Although the result of Theorem 3.1 was taken from [6], no proof has been given in [6]. Thus, for the sake of convenience of the reader, we shall provide the proof of Theorem 3.1 in Appendix A.

Furthermore, by assuming that the initial state $x_{0}$ is a random vector uniformly distributed on the surface of the $n$-dimension unit sphere, the performance (7) can be reduced to

$$
\bar{J}=1 /(2 n) \operatorname{trace}\left(K_{i_{0}}\right) .
$$


The performance degradation of $\bar{J}$ with respect to the optimal performance under full state feedback $J^{*}$ can be measured by the degree of suboptimality $\mu$, where

$$
\mu=\left(\bar{J}-J^{*}\right) / J^{*}
$$

From (12) and (11), it is clear that this suboptimality $\mu$ depends on the initial plant mode $r\left(t_{0}\right)$. By assuming that $r\left(t_{0}\right)$ has a uniform distribution, the average value for $\mu$ can be found. More precisely, if

$$
P_{0 i}=\operatorname{Prob}\left(r\left(t_{0}\right)=i\right)=1 / N
$$

an average suboptimal criterion is defined by

$$
\bar{J}=\frac{1}{2 N n} \sum_{i=1}^{N} \operatorname{trace}\left(K_{i}\right)
$$

Furthermore, a sufficient condition for $\bar{A}_{i}, i=1, \ldots, N$ to be stable is that

$$
\Lambda_{i}=\frac{1}{2}\left[-\bar{A}_{i}-\bar{A}_{i}^{\top}\right]
$$

be positive definite. Thus, by Sylvester's criterion, it is necessary and sufficient to ensure that for each $i=1, \ldots, N$, the determinants of all the principle minors of $\Lambda_{i}$ are greater than or equal to $\varepsilon>0$, where $\varepsilon$ is some small positive number. Let the determinants of the $n$ principle minors of $\Lambda_{i}$ be denoted by $g_{i, 1}, \ldots, g_{i, n}$ respectively. Then we have

$$
g_{i, j} \geq \varepsilon, \quad j=1, \ldots, n .
$$

Thus, we obtain a deterministic optimization problem (P) as follows:

$$
(P)\left\{\begin{array}{l}
\min \bar{J}=\frac{1}{2 N n} \sum_{i=1}^{N} \operatorname{trace}\left(K_{i}\right) \\
\text { subject to } g_{i j} \geq \varepsilon, \quad i=1, \ldots, N ; j=1, \ldots, n
\end{array}\right.
$$

where $K_{i}$ are the solutions of (8). The problem (P) can be easily solved by any existing nonlinear programming software, such as NLQP.

In order to solve the problem (P), we need to calculate the gradient of $\bar{J}$ and $g_{i, j}$ with respect to the gain matrix $F_{i}$. The method for calculating these gradients is given in Section 4. 


\section{Gradient of the cost functional and the constraints}

To derive a formula for the gradient of the cost function $\bar{J}$ and the constraints $g_{i j}$, we need to regard (8) as the state equation in the optimal control problem. We first introduce the Hamiltonian $H$ and the co-state matrices $\lambda_{k}(k=1, \ldots, N)$ for $\bar{J}$. Let

$$
\begin{aligned}
H= & \frac{1}{2 N n} \sum_{i=1}^{N} \operatorname{trace}\left\{K_{i}\right\} \\
& +\sum_{i=1}^{N} \operatorname{trace}\left\{\lambda_{i}\left[K_{i} \tilde{A}_{i}+\tilde{A}_{i}^{\top} K_{i}+Q+C_{i}^{\top} F_{i}^{\top} R F_{i} C_{i}+\sum_{j=1}^{N} \pi_{i j} K_{j}\right]\right\},
\end{aligned}
$$

where the co-state matrices $\lambda_{k}(k=1, \ldots, N) \in R^{n \times n}$ are symmetric, satisfying the following adjoint system:

$$
\frac{\partial H}{\partial K_{k}}=0 \quad \text { implies } \quad \frac{1}{2 N n} I+\lambda_{k} \tilde{A}_{k}^{\top}+\tilde{A}_{k} \lambda_{k}+\sum_{i=1}^{N} \pi_{i k}^{T} \lambda_{i}=0 .
$$

THEOREM 4.1. The gradient of $\bar{J}$ is given by

$$
\begin{aligned}
\frac{\partial \vec{J}}{\partial F_{i}} & =\frac{\partial H}{\partial F_{i}} \\
& =2 R F_{i} C_{i} \lambda_{i} C_{i}^{\top} \quad(i=1, \ldots, N) .
\end{aligned}
$$

The proof of this theorem will be given in Appendix B.

REMARK 4.1. To calculate $\partial \bar{J} / \partial F_{i}$, we need to solve the system comprising of (8) and (19). This system is known as a system of Lyaponuv equations, which can be solved by existing software.

REMARK 4.2. The method for calculating the gradients of the constraints is exactly the same as that for calculating the gradients of $\bar{J}$.

\section{Illustrative example}

EXAMPLE 5.1. (Same as the example given in [6, page 899].)

Let $x \in R^{2}, u \in R, y \in R$. There are two modes, with the transition matrix $\Pi=\left(\begin{array}{cc}0 & 0 \\ 1 & -1\end{array}\right)$. In mode 1 , the plant is governed by

$$
A_{1}=\left(\begin{array}{cc}
-1 & 0.5 \\
0 & 1
\end{array}\right), \quad B_{1}=\left(\begin{array}{l}
0 \\
1
\end{array}\right), \quad C_{1}=(0,1)
$$


In mode 2, the plant is governed by

$$
A_{2}=\left(\begin{array}{cc}
0.25 & 0.5 \\
0 & 0.25
\end{array}\right), \quad B_{2}=\left(\begin{array}{l}
0 \\
1
\end{array}\right), \quad C_{2}=(0,1) .
$$

The criterion (3) is considered with unity weighting matrices $Q$ and $R$.

From [6], we know that the optimal solution with full state feedback is given by:

$$
\begin{aligned}
& K_{1}=\left(\begin{array}{ll}
0.4949 & 0.1010 \\
0.1010 & 2.4495
\end{array}\right) \quad \text { and a gain matrix } \quad F_{1}=\left(\begin{array}{ll}
0.1010 & 2.4495
\end{array}\right) \text {; } \\
& K_{2}=\left(\begin{array}{cc}
2.3548 & 0.5635 \\
0.5635 & 1.768
\end{array}\right) \quad \text { and a gain matrix } \quad F_{2}=\left(\begin{array}{ll}
0.5635 & 1.7688
\end{array}\right) \text {. }
\end{aligned}
$$

The technique employed in [6], which is based on the necessary condition of optimality, yields the following result:

$$
\begin{aligned}
& K_{1}=\left(\begin{array}{cc}
0.5 & 0.0995 \\
0.0995 & 2.4503
\end{array}\right) \quad \text { and a gain matrix } \quad F_{1}=2.5135 \text {; } \\
& K_{2}=\left(\begin{array}{cc}
3.0 & 0.6577 \\
0.6577 & 1.7967
\end{array}\right) \quad \text { and a gain matrix } \quad F_{2}=1.9319 \text {. }
\end{aligned}
$$

The results obtained by solving the approximate problem $(\bar{P})$ is as follow:

$$
\begin{aligned}
& K_{1}=\left(\begin{array}{cc}
0.5 & 0.1010 \\
0.1010 & 2.4497
\end{array}\right) \quad \text { and a gain matrix } \quad F_{1}=2.4749 ; \\
& K_{2}=\left(\begin{array}{cc}
3.0 & 0.6583 \\
0.6583 & 1.7967
\end{array}\right) \quad \text { and a gain matrix } \quad F_{2}=1.9319 \text {. }
\end{aligned}
$$

Let $J^{*}, J^{0}$ and $J^{1}$ be the value of the objective function which corresponds to the full state feedback solution, the optimal output feedback obtained by Mariton and Bertrand [6] and the optimal output feedback obtained by our method respectively. Let $\mu^{0}$ and $\mu^{1}$ be the value of $\mu$ obtained by substituting $J=J^{0}$ and $J=J^{1}$ in (12) respectively. The performance can be summed up in Table 5.1, depending on the initial mode distribution

$$
P_{0}=\left(\begin{array}{c}
P_{01} \\
1-P_{01}
\end{array}\right) \text {. }
$$

From the values of $\mu^{0}$ and $\mu^{1}$ in Table 5.1, it is clear that the expected performances obtained by our method is slightly better than those obtained by Mariton and Bertrand [6].

When $P_{01}=1, r\left(t_{0}\right)=1$, the result obtained by our method is $0.02 \%$ better than that obtained in [6]. When $P_{01}=0.5, \operatorname{Prob}\left(r\left(t_{0}\right)=1\right)=0.5$, the result obtained 
TABLE 5.1. Comparison of optimal solution

\begin{tabular}{|c|c|c|c|}
\hline$P_{01}$ & 1 & 0.5 & 0 \\
\hline $8 \times J^{*}$ & 2.9444 & 3.6309 & 4.3168 \\
\hline $8 \times J^{0}$ & 2.9503 & 3.8735 & 4.7967 \\
\hline $8 \times J^{1}$ & 2.9497 & 3.8732 & 4.7967 \\
\hline$\mu^{0}(\%)$ & 0.20 & 6.68 & 11.12 \\
\hline$\mu^{1}(\%)$ & 0.18 & 6.67 & 11.12 \\
\hline
\end{tabular}

by our method is $0.01 \%$ better than that obtained in [6]. When $P_{01}=0, r\left(t_{0}\right)=2$, the accuracy of the result obtained by our method and that obtained in [6] is exactly the same. However, for both our method and that used in [6], the worst performance $\left(P_{01}=0, r\left(t_{0}\right)=2\right)$ using output feedback (5) is only about $10 \%$ worse than the optimal expected performance using full state feedback.

\section{Appendix A}

ProOF OF THEOREM 3.1. From (3), (5), (1b), we get

$$
\begin{gathered}
\bar{J}=E\left\{\frac{1}{2} \int_{t_{0}=0}^{\infty} x(\tau)^{\top}\left[Q+C(r(\tau))^{\top} F(r(\tau))^{\top} R F(r(\tau)) C(r(\tau))\right] x(\tau) d \tau\right. \\
\left.\mid t_{0}, x\left(t_{0}\right), r\left(t_{0}\right)\right\} .
\end{gathered}
$$

In view of (8), (A1) can be simplified to

$$
\begin{aligned}
& \bar{J}=E\left\{\frac{1}{2} \int_{t_{0}=0}^{\infty}-x(\tau)^{\top}\left[\tilde{A}(r(\tau))^{\top} K(r(\tau))+\right.\right. \\
&\left.\left.K(r(\tau)) \tilde{A}(r(\tau))+\sum_{j=1}^{N} \pi_{r(\tau), j} K(r(\tau))\right] x(\tau) d \tau \mid t_{0}, x\left(t_{0}\right), r\left(t_{0}\right)\right\} .(\mathrm{A} 2)
\end{aligned}
$$

Now let

$$
V(t)=x(t)^{\top} K(r(t)) x(t)
$$

and

$$
V_{i}(t)=E\{V(t) \mid r(t)=i\} .
$$

Then, from (1a), (5), (1b) and (9), we have

$$
\dot{x}(t)=\tilde{A}(r(t)) x(t) .
$$


Thus, by differentiating (A4), we get

$$
\dot{V}_{i}(t)=E\left\{x(t)^{\top}\left[\tilde{A}(r(t))^{\top} K(r(t))+K(r(t)) \tilde{A}(r(t))+\dot{K}(r(t))\right] x(t) \mid r(t)=i\right\} .
$$

On the other hand,

$E\{\dot{K}(r(t)) \mid r(t)=i\}=\lim _{\Delta \rightarrow 0+} \frac{E\{K(r(t+\Delta)) \mid r(t)=i\}-E\{K(r(t)) \mid r(t)=i\}}{\Delta}$.

But

$$
\lim _{\Delta \rightarrow 0+} E\{K(r(t+\Delta)) \mid r(t)=i\}=K_{i}+\Delta \sum_{j=1}^{N} \pi_{i j} K_{j}+0(\Delta),
$$

so that (A7) becomes

$$
E\{\dot{K}(r(t)) \mid r(t)=i\}=\sum_{j=1}^{N} \pi_{i j} K_{j}
$$

Thus, from (A6), (A9), we get

$$
\dot{V}_{i}(t)=E\left\{x(t)^{\top}\left[\tilde{A}(r(t))^{\top} K(r(t))+K(r(t)) \tilde{A}(r(t))+\sum_{j=1}^{N} \pi_{i j} K_{j}\right] x(t) \mid r(t)=i\right\} .
$$

From (A2) and (A10), we get

$$
\begin{aligned}
\bar{J} & =E\left[\frac{1}{2} \int_{t_{0}=0}^{\infty}-\dot{V}(t) d t \mid t_{0}, x\left(t_{0}\right) r\left(t_{0}\right)\right] \\
& =\frac{1}{2}\left[x_{0}^{\top} K_{i_{0}} x_{0}-E\left(V(\infty) \mid t_{0}, x\left(t_{0}\right), r\left(t_{0}\right)\right)\right] .
\end{aligned}
$$

Since $\tilde{A}_{i}+\frac{1}{2} \pi_{i i} I$ is stable for all $i$, we have

$$
E\left\{V(\infty) \mid t_{0}, x\left(t_{0}\right), r\left(t_{0}\right)\right\}=0
$$

The conclusion of this theorem follows easily from (A11) and (A12).

\section{Appendix B}

PROOF OF THEOREM 4.1. Let $F_{i}$ be any parameter matrix when $r(t)=i$ and let $\Delta F_{i}$ be any perturbation about $F_{i}$. Define

$$
F_{i}(\varepsilon)=F_{i}+\varepsilon \Delta F_{i}
$$


For brevity, let $K_{k}(\cdot)$ and $K_{k}(\cdot, \varepsilon)(k=1, \ldots, N)$ denote, respectively, the solutions of $(8)$ corresponding to parameter matrices $\left(F_{1}, \ldots F_{i}, \ldots F_{N}\right)$ and the perturbed parameter matrices $\left(F_{1}, \ldots F_{i-1}, F_{i}+\varepsilon \Delta F_{i}, F_{i+1} \ldots, F_{N}\right)$ respectively. Let

$$
\Delta K_{k}=\left.\frac{\mathrm{d} K_{k}(\cdot, \varepsilon)}{\mathrm{d} \varepsilon}\right|_{\varepsilon=0}, \quad(k=1 \ldots, N) .
$$

Then, from (17), (19) and (8), we get

$$
\begin{aligned}
\Delta \bar{J} & =\left.\frac{\mathrm{d} \bar{J}\left(F_{i}(\varepsilon)\right)}{\mathrm{d} \varepsilon}\right|_{\varepsilon=0} \\
& =\Delta H \\
& =\left\langle\frac{\partial H}{\partial F_{i}}, \Delta F_{i}\right\rangle+\sum_{k=1}^{N}\left\langle\frac{\partial H}{\partial K_{k}}, \Delta K_{k}\right\rangle,
\end{aligned}
$$

where the inner product of two $r_{1} \times r_{2}$ matrices

$$
A=\left(a_{k l}\right) \quad \text { and } \quad B=\left(b_{k l}\right)
$$

is defined by

$$
\langle A, B\rangle=\sum_{l=1}^{r_{2}} \sum_{k=1}^{r_{1}} a_{k l} b_{k l} .
$$

Hence from (19) and (B3), we get

$$
\Delta \bar{J}=\left\langle\frac{\partial H}{\partial F_{i}}, \Delta F_{i}\right\rangle .
$$

Since $\Delta F_{i}$ is arbitrary, the conclusion of the theorem follows readily from (B5).

\section{References}

[1] M. Athans, "The matrix maximum principle”, J. Inform. Control 11 (1967) 592-606.

[2] J. J. Florentin, "Optimal control of continuous - time, Markov, stochastic systems", J. Electron. Control 10 (1961) 473-488.

[3] N. N. Krasovskii and E. A. Lidskii, "Analytic design of controllers in systems with random attributes", J. Automat. Remote Control 22 (1961) 1021-1025, 1141-1146, 1289-1294.

[4] W. S. Levine and M. Athans, "On the determination of the optimal constant output feedback gains for linear multivariable systems", IEEE Trans. Automat. Control AC-15 (1970) 44-48.

[5] M. Mariton, "On the influence of noise on jump linear systems", IEEE Trans. Automat. Control AC-32 (1987) 1094-1097. 
[6] M. Mariton and P. Bertrand, "Output feedback for a class of linear system with stochastic jump parameters", IEEE Trans. Automat. Control AC-30 (1985) 898-900.

[7] P. J. McLane, "Optimal stochastic control of linear systems with state and control dependent disturbances", IEEE Trans. Automat. Control AC-16 (1971) 793-798.

[8] D. D. Sworder, "Feedback control of a class of linear systems with jump parameters", IEEE Trans. Automat. Control AC-14 (1969) 9-14.

[9] W. M. Wonham, "On a matrix Riccati equation of stochastic control", SIAM J. Control Optim. 6 (1968) 681-697.

[10] W. M. Wonham, "Random differential equations in control theory", in Prob. methods in applied maths. 2 (ed. A. T. Bharucha-Reid), (Academic, New York, 1970), 131-212. 